\title{
On the Evolution of a Large Class of Inhomogeneous Scalar Field Cosmologies
}

\author{
J. Ibáñez and I. Olasagasti \\ Dpto. Física Teórica, Universidad del País Vasco \\ Apdo 64448080 Bilbao, Spain.
}

June 24, 2021 


\begin{abstract}
The asymptotic behaviour of a family of inhomogeneous scalar field cosmologies with exponential potential $V=\Lambda e^{k \phi}$ is studied. By introducing new variables we can perform an almost complete analysis of the evolution of these cosmologies. Unlike the homogeneous case (Bianchi type solutions), when $k^{2}<2$ the models do not isotropize due to the presence of the inhomogeneities.
\end{abstract}




\section{Introduction}

In the last years there has been a renewed interest in studying scalar field cosmological solutions mainly due to the prominent role that scalar fields would play in the early stages of the universe driving a period of inflationary expansion. Scalar fields can be thought as arising from the the low-energy limit of string theory or as coming from the dimensional reduction in KaluzaKlein models.

One of the most interesting questions that has been analysed is the socalled cosmic no-hair conjecture which states that the dynamics of a scalar field with a realistic potential could isotropize the space time smoothing out the inital anisotropies and inhomogeneities, giving at late times a FRW universe. Initially, the cosmic no-hair conjecture was proved for space times with a cosmological constant [1]. Since during the "slow-rolling" epoch the scalar field behaves as a cosmological constant, that result was supposed to be applicable to scalar field cosmologies. However, it was soon realised that the picture would change when the dynamics of the scalar field is taken into account [2]. The study of the evolution of cosmological models with scalar field has been done mainly for homogenous solutions (Bianchi type) and, in particular, when the potential of the field is of Liouville type (exponential potential) [3]. Exponential potentials arise in dimensional reduction theories and it is well known that they lead to power law inflation in FRW models.

Although there have been several attempts to study the evolution of the scalar field in inhomogeneous models [4], there is a lack of results concerning how spatial inhomogeneities affect the dynamics of the scalar field. It was shown for a particular family of exact inhomogeneous solutions with exponential potential that there are cases for which the scalar field does not guarantee that the models inflate or isotropize [5]. Since a generic relativistic solution near the initial singularity is neither homogeneous nor isotropic it is worth analysing the effect of spatial inhomogeneities on the isotropization.

In a previous paper (hereafter Paper I) [6] we started the study of the asymptotic behaviour of a particular class of inhomogeneous metrics described by a diagonal $G_{2}$ line-element. In that work we analysed a particular family of $G_{2}$ self-similar solutions with exponential potential. The main conclusions were that the asymptotic behaviour of the model does not depend on the parameters describing the spatial inhomogenity but rather on the parameter $k$, associated with the slope of the exponential potential and the 
behaviour, essentially, was the same as that found for homogeneous solutions, i.e: when $k^{2}<2$ the models isotropized. It was surmised that these conclusions could be explained in terms of the "weak" spatial inhomogeneity and that the introduction of more "strong" inhomogeneities could result in a different behaviour with respect to the homogeneous case. Since our analysis is based only on the study of the equilibrium points, the conclusions we draw have to be considered at the conjecture level, until a more complete analysis of the structure of the field equations for $G_{2}$ metrics is done.

In this paper we extend the analysis of Paper I to a more general class of $G_{2}$ exponential potential cosmologies and we show how in those cases for which the homogeneous solutions isotropized (when $k^{2}<2$ ), here the inhomogeneities prevent the isotropization. Since our analysis is based only on the study of the equilibrium points, the conclusions we draw have to be considered at the conjecture level, until a more complete analysis of the structure of the field equations for $G_{2}$ metrics is achieved.

It is known that scalar field homogeneous cosmological solutions with an exponential potential evolve towards self-similar solutions and it was conjectured that the same is true for $G_{2}$ solutions [7]. In this paper we prove that the asymptotic metrics of the class of solutions studied here are self-similar.

The plan of the paper is the following: in Section 2 we will describe the metric and the assumptions we make in order to simplify the equations. A new set of variables is introduced in such a way that the phase-space of the system is bounded for most of the cases considered. In Section 3 the equilibrium points of the system and the correponding solutions are studied. Finally the conclusions are explained in Section 4.

\section{The metric and the dynamical systems}

In this paper we shall be considering spatially inhomogeneous $G_{2}$ cosmologies admitting a two-parameter abelian group of isometries for which the line element is written as:

$$
d s^{2}=e^{F}\left(-d t^{2}+d z^{2}\right)+G\left(e^{p} d x^{2}+e^{-p} d y^{2}\right),
$$

with all functions appearing in the metric depending on both variables $t$ and $z$. In paper I we considered that the transitivity surface function $G$ was a homogeneous function, depending only on $t$, and that the rest of the metric 
functions were separable. In the present case we would like to extend that analysis allowing the function $G$ to depend on the variable $z$ as well, keeping the separability of the metric functions.

The local behaviour of the models described by metrics of the form (1) is determined by the gradient of the so-called transitivity surface function $G$ [8]. A globally null or spacelike gradient of this function corresponds to plane or cylindrical gravitational waves. If the gradient is globally timelike, however, the metric describes a cosmological model with spacelike singularities and if the character of the gradient changes throughout space time it describes colliding gravitational waves or cosmologies with timelike and spacelike singularities.

As the matter source we shall consider a minimally coupled scalar field with exponential potential:

$$
V(\phi)=\Lambda e^{k \phi} . \quad \Lambda \geq 0
$$

For vanishing $k$ this will be equivalent to a cosmological constant term. The corresponding stress-energy tensor will read:

$$
T_{a b}=\phi_{, a} \phi_{, b}-g_{a b}\left[\frac{1}{2} \phi_{, c} \phi^{c}+V(\phi)\right] .
$$

It is well known [9] that this stress-energy tensor is equivalent to a perfect fluid as long as the gradient of the scalar field is timelike.

For the model under consideration the resulting Einstein and Klein-Gordon field equations are:

$$
\begin{gathered}
\ddot{\phi}-\phi^{\prime \prime}+\frac{\dot{G}}{G} \dot{\phi}-\frac{G^{\prime}}{G} \phi^{\prime}+e^{F} \frac{\partial V}{\partial \phi}=0, \\
\frac{\ddot{G}}{G}-\frac{G^{\prime \prime}}{G}=2 e^{F} V(\phi), \\
\ddot{p}-p^{\prime \prime}+\frac{\dot{G}}{G} \dot{p}-\frac{G^{\prime}}{G} p^{\prime}=0, \\
\frac{\dot{G}^{\prime}}{G}-\frac{1}{2} \frac{\dot{G} G^{\prime}}{G^{2}}+\frac{1}{2} \dot{p}^{\prime}-\frac{1}{2} F^{\prime} \frac{\dot{G}}{G}-\frac{1}{2} \dot{F} \frac{G^{\prime}}{G}=-\dot{\phi} \phi^{\prime}, \\
\frac{\ddot{G}}{G}+\frac{G^{\prime \prime}}{G}-\frac{1}{2}\left(\frac{\dot{G}}{G}\right)^{2}-\frac{1}{2}\left(\frac{G^{\prime}}{G}\right)^{2}-\dot{F} \frac{\dot{G}}{G}-F^{\prime} \frac{G^{\prime}}{G}+\frac{1}{2} \dot{p}^{2}+\frac{1}{2} p^{2}=-\dot{\phi}^{2}-\phi^{\prime 2},
\end{gathered}
$$




$$
\ddot{F}-F^{\prime \prime}-\frac{1}{2}\left(\frac{\dot{G}}{G}\right)^{2}+\frac{1}{2}\left(\frac{G^{\prime}}{G}\right)^{2}+\frac{1}{2} \dot{p}^{2}-\frac{1}{2} p^{\prime 2}=-\dot{\phi}^{2}+\phi^{\prime 2} .
$$

We want to find the general behaviour of the models under consideration and, in particular, their behaviour near the initial singularity and at late times. The treatment of the asymptotic behaviour of the inhomogeneous metrics was initiated in [7] by extending the method developed for homogeneous Bianchi type solutions with a perfect fluid [10]. This method is based on the introduction of a new set of adimensional variables constructed from the fluid quantities. For inhomogeneous metrics the new variables satisfy a system of partial differential equations and the equilibrium points are considered as dynamical equilibrium points. Since the source of our metrics, the scalar field, is not globally equivalent to a perfect fluid we can not apply this method and we have to tackle the problem of resolving the asymptotic behaviour of system (4)-(9) in a different way. In paper I we analised a particular family of solutions of the above system. In particular, we considered a family of solutions for which the inhomogeneity was linear and introduced new dynamical variables in such a way that the phase-space was compact and, therefore, we could describe completely the asymptotic behaviour of the system. We will follow the same approach as that already used in paper I. By fixing the spatial dependence of the metric functions we will write this system of partial differential equations as a set of ODE's and we will use standard dynamical system techniques to find out the structure of the phase-space.

To obtain the desired set of ODEs we have made a number of simplifying assumptions on the metric functions:

i) As in [11 we write the scalar field $\phi$ in terms of a new function $\psi$ as:

$$
\phi=-\frac{k}{2} \log (G)+\psi
$$

Substituting (10) into the Klein-Gordon equation (4) we find that $\psi$ satisfies the same equation as function $p$. This allows us to choose the function $\psi$ proportional to $p$ :

$$
\psi(t, z)=m p(t, z)
$$

where $m$ is an arbitrary constant. 
ii) to simplify the equations further we consider separability of the metric functions and in particular we assume the ansatz, already used by different authors [12, 11]:

$$
\begin{gathered}
G(t, z)=T(t) Z(z) \\
F(t, z)=f(t)+f_{1}(z) \\
e^{p(t, z)}=Q(t) Z(z)^{n} .
\end{gathered}
$$

These are ad hoc assumptions, made with the only motivation of simplifying the Einstein equations. They have been suggested by previous works on inhomogeneous metrics with perfect fluid source.

With these assumptions, the two functions depending on the spatial variable $z$, that is, $f_{1}(z)$ and $Z(z)$ are inmediately fixed by the equations. In fact, we see that $\operatorname{Eq}(6)$ separates into:

$$
n \frac{Z^{\prime \prime}}{Z}=\frac{\dot{T}}{T} \frac{\dot{Q}}{Q}+\frac{\ddot{Q}}{Q}-\left(\frac{\dot{Q}}{Q}\right)^{2}=n \epsilon a^{2}, \quad \epsilon=(-1,0,1),
$$

where $n \epsilon a^{2}$ stands for an arbitrary separation constant. Resolving the above equation we get:

$$
Z(z)= \begin{cases}A \cosh (a z)+B \sinh (a z) & \epsilon=1 \\ A z+B & \epsilon=0 \\ A \cos (a z)+B \sin (a z) & \epsilon=-1\end{cases}
$$

where $A$ and $B$ are arbitrary constants of integration.

From Eq.(5) when $\Lambda \neq 0$ or from $\operatorname{Eq}(7)$ when $\Lambda=0$ we find that:

$$
f_{1}(z)=2 \alpha \log Z(z)+\log D \text {. }
$$

When $\Lambda=0, \alpha$ and $D$ are arbitrary constants, but when $\Lambda \neq 0, D$ is still an arbitrary constant but

$$
2 \alpha=C \equiv k^{2} / 2-k m n
$$

The rest of the equations separate in a straightforward way giving rise to new separable constants and a few relations betwen them. We shall not give here the details of the calculations but only summarize them. 
When $Z(z) \neq e^{ \pm a z}$ we obtain from Eq.(8) or from Eq.(9) the following constraint on the parameters:

$$
2 \alpha=n^{2} M^{2}+C-\left(\frac{1}{2}+\frac{k^{2}}{4}\right),
$$

where we have defined $M^{2} \equiv 1 / 2+m^{2}$. When $\Lambda=0$ the above equation fixes the value of $\alpha$, but when $\Lambda \neq 0$ it becomes, from (18),

$$
n^{2} M^{2}=\frac{1}{2}+\frac{k^{2}}{4}
$$

The last relation allows us to write $m$ in terms of $n$ and $k$. The two solutions are:

$$
m_{ \pm}= \pm \frac{\sqrt{k^{2}+2-2 n^{2}}}{2 n}
$$

which also gives $C$ :

$$
C_{ \pm}=\frac{k}{2}\left(k \mp \sqrt{k^{2}+2-2 n^{2}}\right) .
$$

From now on we assume that Eqs(19)-(22) hold. That means that, in the exponential case, we are only considering those solutions for which the constants verify the above relations. In the general exponential case all the solutions are self-similar and the constant $m$ is not restricted by relation (21). Since this case will not add any new insights to the main conclusions of the paper we leave the corresponding analysis to be discussed elsewhere. We are also assuming that $\Lambda \neq 0$. Solutions with $\Lambda=0$ shall be limiting cases from which all the solutions with $\Lambda \neq 0$ start their evolution.

Finally the line element and scalar field have the form:

$$
\begin{gathered}
d s^{2}=D e^{f} Z^{2 \alpha}\left(-d t^{2}+d z^{2}\right)+T Q Z^{(1+n)} d x^{2}+\frac{T}{Q} Z^{(1-n)} d y^{2} \\
\phi=\frac{-k}{2} \log T+m \log Q+(m n-k / 2) \log Z .
\end{gathered}
$$

Where the function $Z(z)$ is defined in (16).

Among the solutions described by the line element (23) we can identify the following known solutions, all of them correponding to the case when 
$\alpha=0$ : If $Z(z)=\sin (a z)$, or $\cos (a z), D e^{f}=Q T$ and $n^{2}=1$, then the solutions reduce to the Kantowski-Sachs models; if $Z(z)=e^{ \pm a z}$, then one obtains that: when $n^{2}=0$ (23) reduces to a type Bianchi V solution, when $n^{2}=1$ to a type Bianchi III and when $n^{2} \neq 0,1$ to type Bianchi VI. Finally, if $Z(z)=$ constant $(23)$ is a type Bianchi I solution.

We can see also from Eq.(24) that when $m n=k / 2$ the scalar field is homogeneous and, therefore, can be cast in a perfect fluid form. In this case the metric (23) belongs to the classes of solutions studied by different authors [11], [12].

Another important class of solutions belonging to the line element (23) is that obtained when $k=m=0$ (from Eq.(20) $n^{2}=1$ ). In this case the scalar field vanishes and the source of the metric is a cosmological constant.

Once the spatial dependence has been fixed, we have to find the time dependence of the metric that is determined by the three independent functions $T(t), Q(t)$ and $f(t)$. We introduce, for later convenience, a new function $h(t)$ defined as:

$$
h(t)=f+k m \log Q .
$$

The Einstein equations, with the above assumptions, reduce to:

$$
\begin{gathered}
\ddot{T}=\epsilon a^{2}+2 \Lambda D \exp \left(h-\frac{k^{2}}{2} \log T\right), \\
\frac{\ddot{Q}}{Q}=n \epsilon a^{2}-\frac{\dot{T}}{T} \frac{\dot{Q}}{Q}+\left(\frac{\dot{Q}}{Q}\right)^{2}, \\
\ddot{h}=\frac{k^{2}}{2} \epsilon a^{2}-M^{2}\left(\frac{\dot{Q}}{Q}\right)^{2}-\left(\frac{k^{2}}{4}-\frac{1}{2}\right)\left(\frac{\dot{T}}{T}\right)^{2}, \\
\frac{\dot{T}}{T}+2 n M^{2} \frac{\dot{Q}}{Q}-\dot{h}=0, \\
\dot{h} \frac{\dot{T}}{T}-M^{2}\left(\frac{\dot{Q}}{Q}\right)^{2}-\left(\frac{k^{2}}{4}-\frac{1}{2}\right)\left(\frac{\dot{T}}{T}\right)^{2}=2 \Lambda D \exp \left(h-\frac{k^{2}}{2} \log T\right)+2 \epsilon a^{2} .
\end{gathered}
$$

From the original system of PDE's we have obtained a system of five ODE's. These equations separate into three dynamical equations and two 
constraint equations. Since equation (26), however, depends on $T$ and its second derivative the associated dynamical system will have four first order equations and two constraint equations. On the other hand, the constraint (30) will serve to eliminate the parameter $\epsilon a^{2}$ from the system.

To put this system of equations in the usual dynamical system form, we make an appropriate change of variables. We want the new variables to be normalized in such a way that they remain bounded near the initial singularity.

From Eq. (30) we see that an appropriate set of new variables $\beta, \Phi$ and $\Gamma$ is:

$$
\beta=\frac{M \frac{\dot{Q}}{Q}}{b \frac{\dot{T}}{T}+d \dot{h}} \quad \Phi=\frac{d \dot{h}}{b \frac{\dot{T}}{T}+d \dot{h}} \quad \Gamma=\sqrt{2 \Lambda D} \frac{\exp \left(\frac{1}{2}\left[h-\frac{k^{2}}{2} \log T\right]\right)}{b \frac{\dot{T}}{T}+d \dot{h}}
$$

with

$$
\begin{aligned}
& b^{2}=\frac{\left|2-k^{2}\right|}{4}, \quad d^{2}=\frac{1}{\left|2-k^{2}\right|} \quad k^{2} \neq 2 \\
& b=d=1 \quad k^{2}=2
\end{aligned}
$$

$\Gamma$ is related with the potential of the scalar field, it vanishes when the scalar field is massless, $\beta$ is related with the time dependent part of the function $p$ in the metric (11) and $\Phi$ depends on $f$. Unlike the Bianchi type solutions, where the kinematical quantities of the source are used as variables, here, since the scalar field is not globally timelike, we can not use that approach. The reason for defining the variables as in (31) is to get a bounded phase space (at least for a large subset of the solutions). Thus there is no particular physical meaning for these variables, although another set of variables with some physical meaning could perhaps be found.

In terms of the new variables (31) and substituting $\epsilon a^{2}$ with the aid of Eq.(30), Eq.(26) becomes:

$$
\frac{\ddot{T}}{T}=\left[\frac{1}{2} b^{2} \frac{\Gamma^{2}-\beta^{2}}{(1-\Phi)^{2}}+\frac{1}{2} \frac{b}{d} \frac{\Phi}{1-\Phi}-\frac{1}{8}\left(k^{2}-2\right)\right]\left(\frac{\dot{T}}{T}\right)^{2} .
$$

To decouple this equation from the rest of equations of the system we define a new time $\tau$, through the following expression:

$$
\dot{\tau}=b \frac{\dot{T}}{T}+d \dot{h}=\frac{b}{1-\Phi} \frac{\dot{T}}{T} .
$$


From the general behaviour near the initial singularity found by Belinskii et al [13], we can assume that our models, for constant $z$, behave near the initial singularity like a Kasner model, in the sense that when $t \rightarrow 0$

$$
T \sim t, \quad f \sim \log Q \sim \phi \sim \log t .
$$

This means that $\dot{\tau} \sim 1 / t$ when $t \rightarrow 0$ which guarantees the regular behaviour of the new variables near the initial singularity. To see how $\tau$ behaves far from the singularity we write Eq.(30) in the following way:

$$
\begin{aligned}
\dot{\tau}^{2}= & 2 \Lambda D \exp \left(h-\frac{k^{2}}{2} \log T\right)+2 \epsilon a^{2}+M^{2}\left(\frac{\dot{Q}}{Q}\right)^{2} \\
& +d^{2} \dot{h}^{2}+\left(b^{2}+\frac{k^{2}-2}{4}\right)\left(\frac{\dot{T}}{T}\right)^{2}
\end{aligned}
$$

when $k^{2} \neq 2$ and

$$
\dot{\tau}^{2}=4 \Lambda D \exp (h-\log T)+4 \epsilon a^{2}+2 M^{2}\left(\frac{\dot{Q}}{Q}\right)^{2}+\dot{h}^{2}+\left(\frac{\dot{T}}{T}\right)^{2}
$$

when $k^{2}=2$.

As long as $\epsilon=0,1$ all the terms in the right hand side of the above two expressions are positive and, therefore, $\dot{\tau}$ is different from zero for all values of $t$. Because of this we can restrict our attention to $\dot{\tau}>0$ which implies that our new time $\tau$ will grow monotonically with $t$. Since $\dot{\tau} \sim 1 / t$ for $t \sim 0$, the range of $\tau$ goes from $-\infty$ to $+\infty$. On the other hand, the situation with $\epsilon=-1$ is completely different. Since there is nothing preventing $\dot{\tau}$ from being zero at some finite time $t$, there will be solutions whose trajectories in the phase-space of the new variables will escape to infinity in a finite time. That means that, in this case, $\tau$ will no longer serve to monitor the evolution in the coordinate time $t$.

We are now in a position to write the equations in terms of these variables. Since the change of variables does not depend on $k$ in a continuous way, the system of equations does not have the same form for all the values of $k$. First we write the constraint equations (29) and (30) that in these new variables take particularly simple expressions. The first constraint equation 
(29) relates $\Phi$ and $\beta$ :

$$
\begin{aligned}
& \Phi=\frac{2+\sqrt{4-k^{4}} \beta}{4-k^{2}} \quad k^{2}<2 \\
& \Phi=\beta+\frac{1}{2} \quad k^{2}=2 \\
& \Phi=\frac{2+\sqrt{k^{4}-4} \beta}{k^{2}} \quad k^{2}>2 .
\end{aligned}
$$

As for the second constraint Eq.(30) it takes the simple form:

$$
L(\beta, \Phi, \Gamma) \equiv 1-\beta^{2}-\Phi^{2}-\Gamma^{2}+H(\beta, \Phi, \Gamma)=\frac{2 \epsilon a^{2}}{\dot{\tau}^{2}}
$$

where

$$
\begin{cases}H=0 & k^{2}<2 \\ H=\Phi-1 & k^{2}=2 \\ H=-2(\Phi-1)^{2} & k^{2}>2\end{cases}
$$

Finally, substituting (31) in Eqs.(26)-(28) we get the dynamical equations for the different ranges of the parameter $k$

$k^{2}<2$ :

$$
\begin{aligned}
\beta^{\prime} & =\left(\frac{1}{2} \sqrt{1-b^{2}}-\frac{3}{4 b} \beta\right) L-\frac{2 b^{2}+1}{2 b} \beta \Gamma^{2} \\
\Phi^{\prime} & =\frac{1}{4 b}\left[\left(3-3 \Phi-2 b^{2}\right) L+2\left[1-\left(2 b^{2}+1\right) \Phi\right] \Gamma^{2}\right] \\
\Gamma^{\prime} & =\frac{\Gamma}{4 b}\left[-3 L+2\left(2 b^{2}+1\right)\left(1-\Gamma^{2}\right)-2 \Phi\right]
\end{aligned}
$$

$$
k^{2}=2
$$

$$
\begin{aligned}
\beta^{\prime} & =\frac{1}{2}(1-4 \beta) L-2 \beta \Gamma^{2} \\
\Phi^{\prime} & =\frac{1}{2}(3-4 \Phi) L+(1-2 \Phi) \Gamma^{2} \\
\Gamma^{\prime} & =-\Gamma\left(2 L-\frac{1}{2}+2 \Gamma^{2}\right)
\end{aligned}
$$




$$
\begin{aligned}
k^{2} & >2: \\
\beta^{\prime} & =\left(\frac{1}{2} \sqrt{1+b^{2}}-\frac{3+4 b^{2}}{4 b} \beta\right) L-\frac{1}{2 b} \beta \Gamma^{2}\left(1+2 b^{2}\right) \\
\Phi^{\prime} & =\frac{1}{4 b}\left(\left[\left(3+4 b^{2}\right)(1-\Phi)-2 b^{2}\right] L+2\left[1-\left(1+2 b^{2}\right) \Phi\right] \Gamma^{2}\right) \\
\Gamma^{\prime} & =-\frac{\Gamma}{4 b}\left[\left(3+4 b^{2}\right) L-2\left(1-2 b^{2}\right)+2\left(1-4 b^{2}\right) \Phi+2\left(1+2 b^{2}\right) \Gamma^{2}\right] .
\end{aligned}
$$

The prime stands for the derivative with respect to the new time $\tau$ and $L$ is defined in (39).

From (39)-(40) we see that there are three different invariant subspaces,

one for each value of the parameter $\epsilon$. The first is the closed surface $L=0$ which corresponds to the linear case $\epsilon=0$. The other two subspaces are separated by the surface $L=0$. The invariant subspace for $\epsilon=1$ is a compact region limited by the surfaces $L=0$ and $\Gamma=0$ and, finally, the invariant subspace for $\epsilon=-1$ is the unbounded region outside $L=0$.

The most satisfactory scenario to give the explicit form of the asymptotic behaviour of the models is the one in which we have variables which are bounded for all times. This is guaranteed for both $\epsilon=0$ and $\epsilon=1$ since we have seen that in these two cases $\dot{\tau}$ remains always positive and the phasespace is bounded. As explained above this is not the case for $\epsilon=-1$. We will see from the numerical calculation of the trajectories that, as expected, for $\epsilon=-1$ some trajectories go to infinity in a finite time. Probably one can find a different set of variables for which this part of the phase space becomes bounded, although it is unlikely that this can be done for the whole phase-space.

\section{The equilibrium points and the associated metrics}

We are interested in looking for results concerning no-hair theorems, thus for each of these dynamical systems we will look for the equilibrium points and their stability. This is important since attractors in phase-space will be related to the behaviour of the models in the limit $\tau \rightarrow \infty(t \rightarrow \infty)$ while 
sources, the unstable fixed points, will be related to the behaviour near the initial singularity $\tau \rightarrow-\infty(t \rightarrow 0)$.

Before giving the explicit expressions of the equilibriun points of the system, it is interesting to investigate whether the equilibrium points represent self-similar solutions. It has been shown that the homogeneous but anisotropic models (Bianchi models) evolve towards self-similar solutions [10] and it has been conjectured that the same behaviour is shared by the $G_{2}$ metrics [7]. We will prove that the solutions considered in this paper do approach self-similar solutions. Here is a sketch of the proof of this statement.

When $\epsilon=0,1$ the equilibrium points are characterized by finite constant values of the variables $\beta, \Phi$ and $\Gamma$, then for an equilibriun point equation (33) takes the form:

$$
\frac{\ddot{T}}{T}=(1-W)\left(\frac{\dot{T}}{T}\right)^{2}
$$

where $W$ is a function both of the constant values of $\beta, \Phi$ and $\Gamma$ and the parameters of the model. This equation has only two types of solutions depending on whether or not $W$ is zero. When $W \neq 0$ the solution is of the form $T=t^{1 / W}$ while when $W=0$ it is an exponential, that is, $T=e^{\bar{W} t}$, where $\bar{W}$ is another constant. For the power law solution $T=t^{1 / W}$, equations (34) and (39) force $\epsilon=0$ which in turn forces $Z(z)$ to be linear in $z$.

Writing down the equations for a homothetic vector $\vec{\xi}$,

$$
£_{\vec{\xi}} g_{a b}=2 g_{a b},
$$

we find that for both solutions, exponential and power law, there is at least one homothetic vector with components $\xi^{a}=\left(a_{0}, a_{1} x, a_{2} y, 0\right)$ in the exponential case and $\xi^{a}=\left(b_{0} t, b_{1} x, b_{2} y, b_{0} z\right)$ in the power law case, where both $a_{m}$ and $b_{m}$ are constants (the details of this calculation are given in the Appendix)

To give a qualitative behaviour of the solutions of the system (41)-(43) it is important to obtain the equilibrium points and their local stability. Let us first note that the system admits the symmetry $\Gamma \rightarrow-\Gamma$. Therefore, the study of the equilibrium points will be restricted to $\Gamma \geq 0$. They can be found explicitely and are given by: 


\section{$P_{1}$ and $P_{2}$}

$$
\begin{array}{lll}
\beta^{2}+\Phi^{2}=1 & \Gamma=0 & k^{2}<2 \\
\beta^{2}+\Phi^{2}-\Phi=0 & \Gamma=0 & k^{2}=2 \\
\beta^{2}+(1-\Phi)(1-3 \Phi)=0 & \Gamma=0 & k^{2}>2
\end{array}
$$

These points lie on the surface $L=0$ corresponding, therefore, to $\epsilon=0$. All of them are the sources of the entire phase-space, i.e: the attractors at early times. This set of points reduces to two separate points when the constraint equation (38) is taken into account. These are the two points given by the intersection in $\Gamma=0$ of the surface $L=0$ and the plane defined by the first constraint equation (38). For $k^{2} \geq 6$ one of them becomes a saddle, attracting the trajectories lying on $L=0$. Since $\Gamma=0$, the corresponding metrics describe massless scalar field cosmologies. The line element and the scalar field are:

$$
\begin{aligned}
d s^{2} & =D t^{C_{1}} E_{z}^{C_{ \pm}}\left(-d t^{2}+d z^{2}\right)+t^{1+C_{2}} E_{z}^{1+n} d x^{2}+t^{1-C_{2}} E_{z}^{1-n} d y^{2} \\
\phi & =\left( \pm C_{2} \frac{\sqrt{k^{2}+2-2 n^{2}}}{2 n}-\frac{k}{2}\right) \log t-\frac{C_{ \pm}}{k} \log E_{z}
\end{aligned}
$$

where

$$
\begin{gathered}
E_{z}=A z+B \\
C_{1}=1+\frac{C_{2}}{n}\left(1+C_{ \pm}\right) \\
C_{2}=n\left(1 \pm \sqrt{\frac{8}{k^{2}+2}}\right) .
\end{gathered}
$$

The - and + signs appearing in the definition of $C_{2}$ correspond to $P_{1}$ and $P_{2}$ respectively. When $n^{2}=1$ and $m n=k / 2 C_{ \pm}=0$ and it is easy to see by a simple coordinate transformation that (46) is a LRS Bianchi type I solution.

$P_{3}$

$$
\begin{array}{llll}
\beta=\frac{2 b \sqrt{1-b^{2}}}{3} & \Phi=1-\frac{2}{3} b^{2} & \Gamma=0 & k^{2}<2 \\
\beta=\frac{1}{4} & \Phi=\frac{3}{4} & \Gamma=0 & k^{2}=2 \\
\beta=\frac{2 b \sqrt{1+b^{2}}}{3+4 b^{2}} & \Phi=\frac{3+2 b^{2}}{3+4 b^{2}} & \Gamma=0 & k^{2}>2
\end{array}
$$


This point corresponds, again, to a massless scalar field but wiht $\epsilon=1$. It is a saddle for all $k$, attracting the trajectories with $\epsilon=1$ in $\Gamma=0$. The metric and the scalar field corresponding to this point are:

$$
\begin{aligned}
d s^{2} & =D e^{\left(2+C_{ \pm}\right) a t} E_{z}^{C_{ \pm}}\left(-d t^{2}+d z^{2}\right)+\left(e^{a t} E_{z}\right)^{1+n} d x^{2}+\left(e^{a t} E_{z}\right)^{1-n} d y^{2} \\
\phi & =-\frac{C_{ \pm}}{k}\left(a t+\log E_{z}\right)
\end{aligned}
$$

where

$$
E_{z}=A \cosh a z+B \sinh a z
$$

and $A$ and $B$ are arbitrary constants.

By making the change: $T=e^{a t} E_{z}, Z=e^{a t} E_{z}^{\prime}$ when $A>B$ we see that (47) is an anisotropic Bianchi type I space time. (For $A \leq B$ the model has spacelike curvature singularities). In the special case when $n^{2}=1$ and $m n=k / 2$ this turns out to be Minkowski flat metric.

$P_{4}$

$$
\begin{array}{llll}
\beta=0 & \Phi=\frac{1}{1+2 b^{2}} & \Gamma=\frac{2 b \sqrt{1+b^{2}}}{1+2 b^{2}} & k^{2}<2 \\
\beta=0 & \Phi=\frac{1}{1+2 b^{2}} & \Gamma=\frac{2 b \sqrt{1-b^{2}}}{1+2 b^{2}} & k^{2}>2
\end{array}
$$

For this point $\epsilon=0$. It does not exist for $k^{2}=2$ or $k^{2} \geq 6$. For $k^{2}<2$ this is the attractor for trajectories with $\epsilon=0$ and $\epsilon=1$ (also for some of the trajectories with $\epsilon=-1$ ). For $2<k^{2}<6$ it is a saddle, attracting only the trajectory with $\epsilon=0$. In this case:

$$
\begin{aligned}
d s^{2} & =\left[\frac{k^{2}-2}{2 \sqrt{6-k^{2}}} t\right]^{\frac{4}{k^{2}-2}}\left[\frac{E_{z}^{C_{ \pm}}}{2 \Lambda}\left(-d t^{2}+d z^{2}\right)+E_{z}^{1+n} d x^{2}+E_{z}^{1-n} d y^{2}\right] \\
\phi & =\frac{2 k}{2-k^{2}} \log \left[\frac{k^{2}-2}{2 \sqrt{6-k^{2}}} t\right]-\frac{C_{ \pm}}{k} \log E_{z}, \quad E_{z}=A z+B
\end{aligned}
$$

When $n^{2}=1$ and $m n=k / 2\left(C_{ \pm}=0\right)$ it is easy to see that this is a flat FRW space time. 
$P_{5}$

$$
\begin{array}{llll}
\beta=\frac{2 b^{3}}{\sqrt{1-b^{2}}} & \Phi=1-2 b^{2} & \Gamma=\frac{2 b}{\sqrt{1-b^{2}}} & k^{2}<2 \\
\beta=0 & \Phi=\frac{1}{2} & \Gamma=\frac{1}{2} & k^{2}=2 \\
\beta=\frac{2 b^{3} \sqrt{1+b^{2}}}{1+5 b^{2}+4 b^{4}} & \Phi=\frac{1+2 b^{2}}{1+4 b^{2}} & \Gamma=\frac{2 b \sqrt{1+b^{2}}}{1+5 b^{2}+4 b^{4}} & k^{2}>2
\end{array}
$$

The solution corresponding to this point has $\epsilon=-1$ when $k^{2}<2, \epsilon=0$ when $k^{2}=2$ and $\epsilon=1$ when $k^{2}>2$. It is a saddle for $k^{2} \leq 2$ and a sink for $k^{2}>2$. When $k^{2}>2$ this is the attractor for the trajectories with $\epsilon=1$ and when $k^{2}=2$ is the attractor for both trajectories with $\epsilon=0$ and $\epsilon=1$. The metric and the scalar field are:

$$
\begin{aligned}
d s^{2}= & \frac{A^{2}-\epsilon a^{2}}{2 \Lambda} e^{C_{1} t} E_{z}^{C_{ \pm}}\left(-d t^{2}+d z^{2}\right) \\
& +e^{\left[\left(A^{2}+n \epsilon a^{2}\right) / A\right] t} E_{z}^{1+n} d x^{2}+e^{\left[\left(A^{2}-n \epsilon a^{2}\right) / A\right] t} E_{z}^{1-n} d y^{2} \\
\phi= & -\frac{1}{k}\left(C_{1} t+C_{ \pm} \log E_{z}\right)
\end{aligned}
$$

where

$$
\begin{gathered}
C_{1}=\frac{k^{2} A^{2} \mp \epsilon a^{2} k \sqrt{k^{2}+2-2 n^{2}}}{2 A}, \quad a^{2}=\epsilon \frac{k^{2}-2}{k^{2}+2} A^{2} \\
E_{z}= \begin{cases}\bar{A} \cosh (a z)+\bar{B} \sinh (a z) & \epsilon=1 \\
\bar{A} z+\bar{B} & \epsilon=0 \\
\bar{A} \cos (a z)+\bar{B} \sin (a z) & \epsilon=-1\end{cases}
\end{gathered}
$$

where $A, \bar{A}$ and $\bar{B}$ are arbitrary constants.

This solution was previously found in [11]. As in the previous cases, when $n^{2}=1$ and $m n=k / 2$ it is homogeneous: when $k^{2}<2$, it is a KantowskiSachs space time; when $k^{2}=2$ it is a flat FRW model while for $k^{2}>2$ it is a LRS Bianchi type III solution. This last case can be seen by making the coordinate transformation:

$$
X=-d x-\ln E_{z}, \quad Z=-\frac{1}{d^{2}} \frac{E_{z}^{\prime}}{E_{z}} e^{-d x}
$$

where $d$ is a constant related with the constants appearing in the solution.

To complete the description of the evolution of the solutions we have solved the system (41)-(43) with the constraint equation (38) by numerical 
integration. The phase-space diagrams so obtained are plotted in Fig.1 for different ranges of the parameter $k$. The bold line represents the invariant subspace $L=0$. This line splits the phase-space into two separate invariant subspaces: the inner region correponds to $\epsilon=1(L>0)$ and the outer region to $\epsilon=-1(L<0)$. The two fixed points $P_{1}$ and $P_{2}$ are located in which constitutes the common points of the two invariant subspaces defined by $L=0$ and $\Gamma=0$ (subspace of massless scalar field solutions). $P_{3}$, lies in the invariant subspace $\Gamma=0, L>0 . P_{4}$ lies in the line $L=0$ and exists only when $k^{2}<2$ and when $2<k^{2}<6$. It aproaches the point $P_{1}$ as $k^{2} \rightarrow 6$ and the point $P_{5}$ as $k^{2} \rightarrow 2$. Finally, $P_{5}$ does not stay in the same invariant subspace for all $k$. As a matter of fact, this point is in the region defined by $L<0$ when $k^{2}<2$, is on $L=0$ when $k^{2}=2$, and lies in $L>0$ when $k^{2}>2$.

\section{Conclusions}

In this work we have considered a class of inhomogeneous solutions with a minimally coupled scalar field with an exponential potential. This class is more general than that studied in paper I. Therefore, we can analyse the effect produced by complex initial inhomogeneities on the dynamics of scalar field cosmologies and specifically on the isotropization of the models.

When the inhomogeneity was linear, as that described in paper I, or when the solutions were homogeneous (Bianchi type), the dynamics of the solutions was completely determined by the value of the parameter $k$ and it was shown that for $k^{2} \leq 2$ all the solutions reached homogeneity and isotropy but when $k^{2}>2$ while all the models still attained homogeneity only a subclass of measure zero reached isotropy.

We have found the equilibrium points of the dynamical systems associated with part of the solutions (23) and we have shown that all of them are selfsimilar. Except the point $P_{3}$, they correspond to inhomogeneous space times with a scalar field configuration that cannot be related to a perfect fluid source. However, in the special case when the scalar field is homogeneous $\left(n^{2}=1\right.$ and $\left.m n=k / 2\right)$, they are all homogeneous perfect fluid space times. The fixed point $P_{3}$ represents a homogeneous perfect fluid space time for every value of the parameters defining the particular model.

We can conclude that although the dynamics is still determined by $k$, as in the former cases, the result refering to the isotropization or homogeneization 
of the space time is completely different. Actually, when $k^{2}<2$ solutions with $\epsilon=0,1$ evolve towards the point $P_{4}$ which is inhomogeneous. Although the point $P_{3}$ is an attractor for a subset of solutions, it is a saddle point, attracting only the massless scalar field solutions. Hence, the inhomogeneities prevent in this case the solutions from isotropizing. Only in the particular case when $n^{2}=1$ and $m n=k / 2$ (corresponding to a homogeneous scalar field) we do have a similar situation to the previous cases. When $k^{2} \geq 2$ the point $P_{5}$ becomes the attractor for the solutions.

In the region where $\epsilon=-1$ the situation is quite different since the variables used are not bounded. If $k^{2}<2$ the stable manifold of the fixed point $P_{5}$ splits this region into two separate regions. The solutions with trajectories taking place in the inner region are attracted by the inhomogeneous fixed point $P_{4}$, whereas those on the stable manifold approach the inhomogeneous solution $P_{5}$. Those with trajectories in the unbounded outer region escape to infinity. When $k^{2} \geq 2$ this separation does no longer exist and all the solutions with $(\epsilon=-1)$ escape to infinity.

Points $P_{1}$ and $P_{2}$ describe the solutions "close" to the initial singularity $t=0$. On both points the potential vanishes since near the singularity the kinematical term is the leading one in the stress-energy tensor. By making a coordinate transformation the line element and the scalar field can be cast into the form:

$$
\begin{aligned}
d s^{2} & =-a_{0}(z) d t^{2}+a_{1}(z) t^{2 p_{1}} d x^{2}+a_{2}(z) t^{2 p_{2}} d y^{2}+a_{3}(z) t^{2 p_{3}} d z^{2} \\
\phi & =q \ln t+b(z)
\end{aligned}
$$

where $p_{i}$ and $q$ are constants verifying

$$
p_{1}+p_{2}+p_{3}=p_{1}^{2}+p_{2}^{2}+p_{3}^{2}+q^{2}=1 .
$$

This agrees with [14] in the sense that the above metric could represent the asymptotic form of a "general" scalar field cosmological solution near the singularity.

It is important to note that solutions corresponding to a cosmological constant can be analysed by letting $k=m=0$. In this case all the solutions evolve to homogeneous space times.

As we mentioned in section 2, in order not to extend this paper the general exponential case will be considered in a separate paper. It is relevant just to note that the asymptotic behaviour in that case is similar to that found here 
in the sense that even when $k^{2}<2$ the equilibrium points of the system are inhomogeneous.

\section{Acknowledgments}

We are grateful to Prof. A.A.Coley and Dr. R.van den Hoogen for stimulating discussions. We are indebted to Prof. A.Feinstein for his comments. This work is supported by a grant DGICYT PB93-0507. I.O.'s work is supported by a fellowship from the DGICYT FP94.

\section{References}

[1] R.M. Wald, Phys. Rev D 28, 2118 (1983); L.G. Jensen and J.A. SteinSchabes, Phys. Rev. D 35, 1146 (1987).

[2] M. Heusler, Phys. Lett. B 253, 33 (1991).

[3] J.J. Halliwell, Phys. Lett B 185, 341 (1987); A.B. Burd and J.D. Barrow, Nucl. Phys. B 308, 929 (1988); Y. Kitada and K. Maeda, Phys. Rev. D 45, 1416 (1992); J. Ibáñez, R.J. Van den Hoogen and A.A. Coley, Phys. Rev. D 51, 928 (1995).

[4] D.S. Goldwirth and T. Piran, Phys. Rev. D 43, 3204 (1991); E.Calzetta and M.Sakallariadou, Phys. Rev. D 45, 2802 (1992).

[5] A.Feinstein and J.Ibáñez, Class. Quantum Grav. 10, L227 (1993); J.M. Aguirregabiria, A.Feinstein and J.Ibáñez, Phys. Rev. D 48, 4669 (1993).

[6] J.Ibáñez and I.Olasagasti, J. Math. Phys. 37,6283 (1996).

[7] C.G. Hewitt and J. Wainwright, Class. Quantum Grav. 7, 2295 (1990).

[8] M. Carmeli, Ch. Charach and S. Malin, Phys. Rep. 76, 79 (1981).

[9] R.R. Tabensky and A.H. Taub, Comm. Math. Phys. 29, 61 (1973).

[10] L. Hsu and J. Wainwright, Class. Quantum Grav. 3, 1103 (1986); J. Wainwright and L.Hsu, Class. Quantum Grav. 6, 1409 (1989).

[11] A.Feinstein, J.Ibáñez and P.Labraga, J. Math. Phys. 36,4962 (1995) 
[12] S.W. Goode, M. Math. thesis, University of Waterloo (1980); J.Wainwright and S.W. Goode, Phys. Rev. D 22, 1906 (1980); E. Ruiz and J.M.M. Senovilla, Phys Rev D 45, 1995 (1992).

[13] V.A.Belinskii, I.M.Khalatnikov and E.M.Lifshitz, Adv. Phys. 19, 525 (1970)

[14] V.A.Belinskii, I.M.Khalatnikov, Zh. Eksp. Teor. Fiz. 57, 2163 (1969) [Sov. Phys.-JETP 30, 1174 (1970)]

\section{A Appendix}

In this appendix we are going to show that all the fixed points of the system under study are indeed self-similar solutions. By inspecting the equilibrium points listed in Section 3 it is easy to see that for the fixed points the functions $e^{f(t)}$ and $Q(t)$ are powers of the function $T(t)$ :

$$
e^{f(t)}=T(t)^{C_{1}} \quad Q(t)=T(t)^{C_{2}},
$$

where $C_{1}$ and $C_{2}$ are constants depending on the equilibrium point we are considering.

The equations for the existence of a homothetic vector $\vec{\xi}$, taking into account Eq.(A.1), reduce to the following equations:

$$
\begin{array}{r}
C_{1} \frac{\dot{T}}{T} \xi^{0}+2 \alpha \frac{Z^{\prime}}{Z} \xi^{3}+2 \partial_{0} \xi^{0}=2 \\
\left(1+C_{2}\right) \frac{\dot{T}}{T} \xi^{0}+(1+n) \frac{Z^{\prime}}{Z} \xi^{3}+2 \partial_{1} \xi^{1}=2 \\
\left(1-C_{2}\right) \frac{\dot{T}}{T} \xi^{0}+(1-n) \frac{Z^{\prime}}{Z} \xi^{3}+2 \partial_{2} \xi^{2}=2 \\
\partial_{0} \xi^{0}=\partial_{3} \xi^{3} \\
D T^{C_{1}} Z^{2 \alpha} \partial_{1} \xi^{0}-T^{1+C_{2}} Z^{1+n} \partial_{0} \xi^{1}=0
\end{array}
$$




$$
\begin{aligned}
D T^{C_{1}} Z^{2 \alpha} \partial_{2} \xi^{0}-T^{1-C_{2}} Z^{1-n} \partial_{0} \xi^{2} & =0 \\
\partial_{3} \xi^{0}-\partial_{0} \xi^{3} & =0 \\
T^{2 C_{2}} Z^{2 n} \partial_{2} \xi^{1}+\partial_{1} \xi^{2} & =0 \\
D T^{C_{1}} Z^{2 \alpha} \partial_{1} \xi^{3}+T^{1+C_{2}} Z^{1+n} \partial_{3} \xi^{1} & =0 \\
D T^{C_{1}} Z^{2 \alpha} \partial_{2} \xi^{3}+T^{1-C_{2}} Z^{1-n} \partial_{3} \xi^{2} & =0
\end{aligned}
$$

We have shown that the function $T(t)$ in the equilibrium points verifies the Eq.(44) giving two different solutions:

I) $W=0 \Rightarrow \frac{\dot{T}}{T}=k=$ constant.

Looking at the equations (A.2)-(A.11) one can try the following solution:

$$
\xi^{0}=\text { constant } \xi^{1}=\xi^{1}(x) \quad \xi^{2}=\xi^{2}(y) \quad \xi^{3}=0 .
$$

Equations (A.5)-(A.11) are automatically satisfied and equations (A.2)(A.4) determine completely $\vec{\xi}$ :

$$
\vec{\xi}=\left\{\frac{2}{C_{1} k}, \frac{C_{1}-C_{2}-1}{C_{1}} x, \frac{C_{1}+C_{2}-1}{C_{1}} y, 0\right\} .
$$

II) $W \neq 0 \Rightarrow \frac{\dot{T}}{T}=\frac{1}{W t}$ and $\frac{Z^{\prime}}{Z}=\frac{1}{z}$

In this case equations (A.2) and (A.5) suggest a solution of the form:

$$
\xi^{0}=c t \quad \xi^{1}=\xi^{1}(x) \quad \xi^{2}=\xi^{2}(y) \quad \xi^{3}=c z,
$$

where $c$ is a constant to be determined.

As before, the actual form is completely determined by equations (A.2)(A.4), the remaining equations being automatically satisfied. Then we obtain:

$$
\begin{aligned}
\vec{\xi}= & \frac{W}{C_{1}+W\left(C_{3}+2\right)}\left\{2 t,\left(\frac{C_{1}-C_{2}-1}{W}+1+C_{3}-n\right) x,\right. \\
& \left.\left(\frac{C_{1}+C_{2}-1}{W}+1+C_{3}+n\right) y, 2 z\right\}
\end{aligned}
$$




\section{Figure Caption}

Figure 1: Phase-space diagrams are drawn for (a) $k^{2}=1$, (b) $k^{2}=2$, (c) $k^{2}=4$ and (d) $k^{2}=10$. Each diagram represents the typical form of the phase-space for the four significant ranges of the parameter $\mathrm{k}$. $\Gamma$ is shown on the vertical axis and $\beta$ on the horizontal axis. The bold line stands for the separatrix $L=0$, the arrows show the direction of increasing time, and the dots represent the fixed points, which are named as in the body of the paper. We can easily distinguish the four important invariant subspaces: $\Gamma=0$ (massless scalar field), $\epsilon=-1$ (the unbounded region outside $L=0$ ), $\epsilon=0$ (the separatrix $L=0$ itself) and $\epsilon=1$ (the bounded region iside $L=0$ ). 


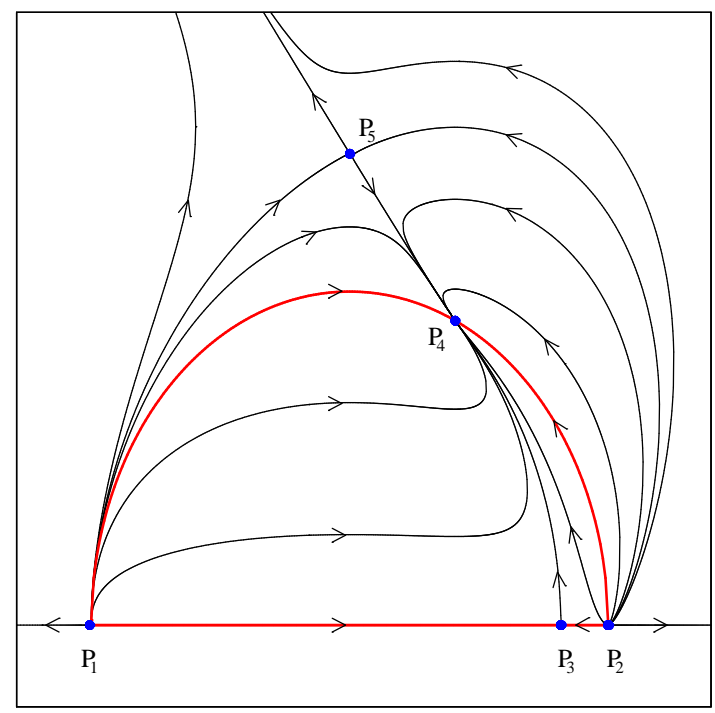

(a) $\mathrm{k}^{2}<2$

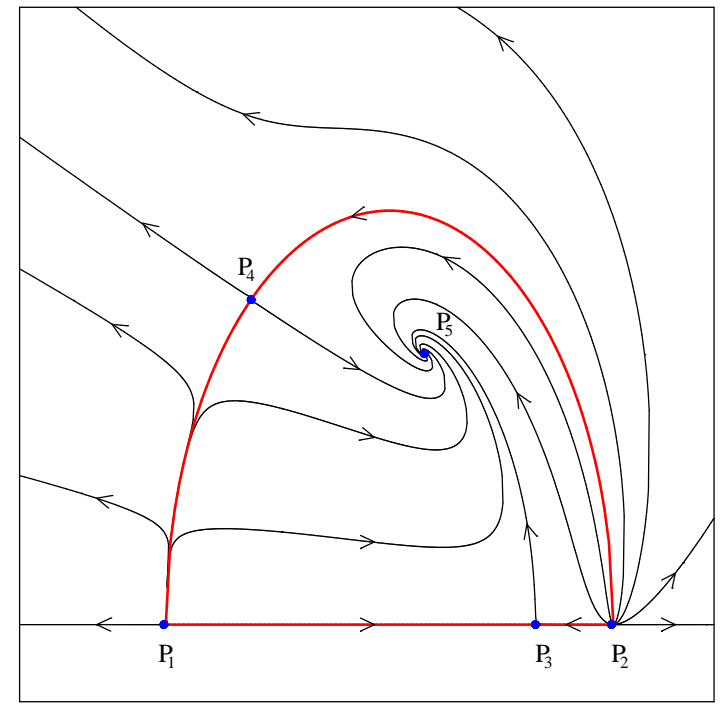

(c) $2<\mathrm{k}^{2}<6$

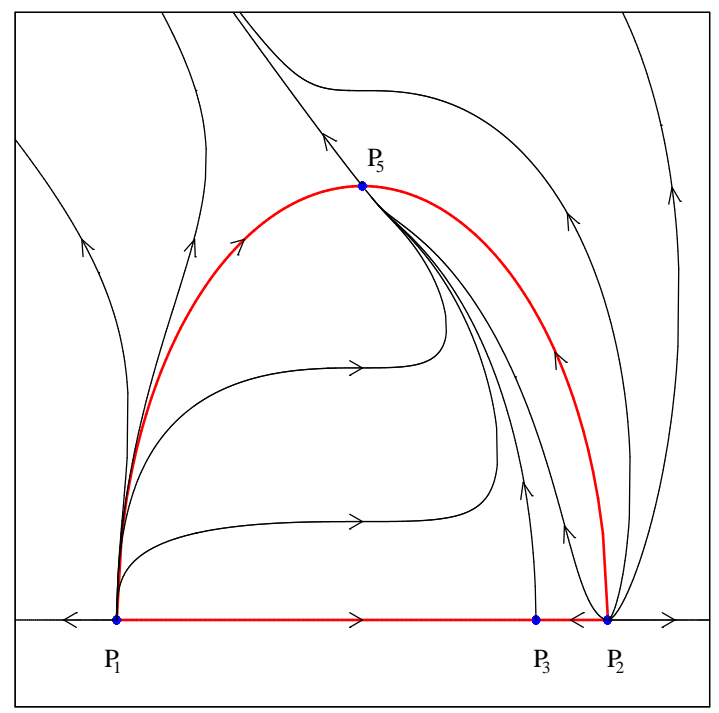

(b) $\mathrm{k}^{2}=2$

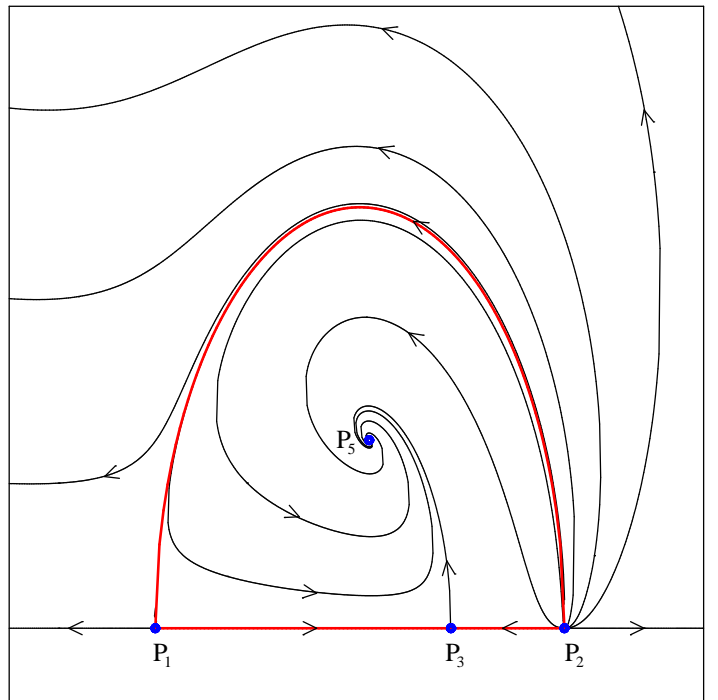

(d) $k^{2}>6$ 